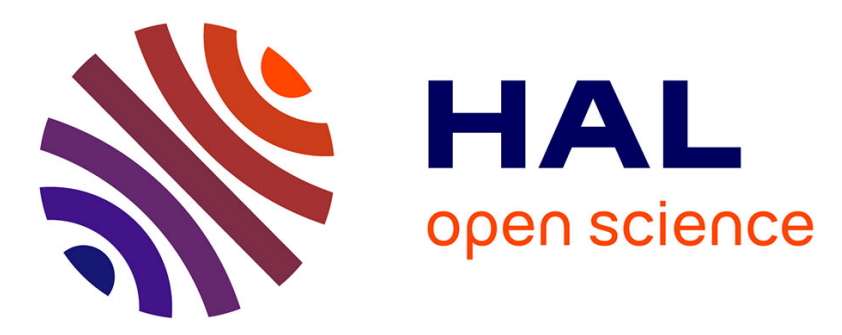

\title{
A Subjective Study of Visibility Thresholds for wavelet domain watermarking
}

Florent Autrusseau, Sylvain David, Vinod Pankajakshan

\section{To cite this version:}

Florent Autrusseau, Sylvain David, Vinod Pankajakshan. A Subjective Study of Visibility Thresholds for wavelet domain watermarking. International Conference on Image Processing, Sep 2010, Hong Kong SAR China. pp.201-204. hal-00495960

\section{HAL Id: hal-00495960 https://hal.science/hal-00495960}

Submitted on 12 Jul 2010

HAL is a multi-disciplinary open access archive for the deposit and dissemination of scientific research documents, whether they are published or not. The documents may come from teaching and research institutions in France or abroad, or from public or private research centers.
L'archive ouverte pluridisciplinaire HAL, est destinée au dépôt et à la diffusion de documents scientifiques de niveau recherche, publiés ou non, émanant des établissements d'enseignement et de recherche français ou étrangers, des laboratoires publics ou privés. 


\section{Cover Page}

1) Title of the paper:

A SUBJECTIVE STUDY OF VISIBILITY THRESHOLDS FOR WAVELET DOMAIN WATERMARKING

2) authors' affiliation and address:

IRCCyN-IVC, (UMR CNRS 6597), Polytech' Nantes

Rue Christian Pauc, La Chantrerie, 44306 NANTES, France.

Tel : 02.40.68.30.52

Fax : 02.40.68.32.32

3) e_mail address:

Florent.Autrusseau@univ-nantes.fr

4) Conference \& Publisher information:

IEEE ICIP 2010

http://www.icip2010.org/

http://www.ieee.org/

5) bibtex entry:

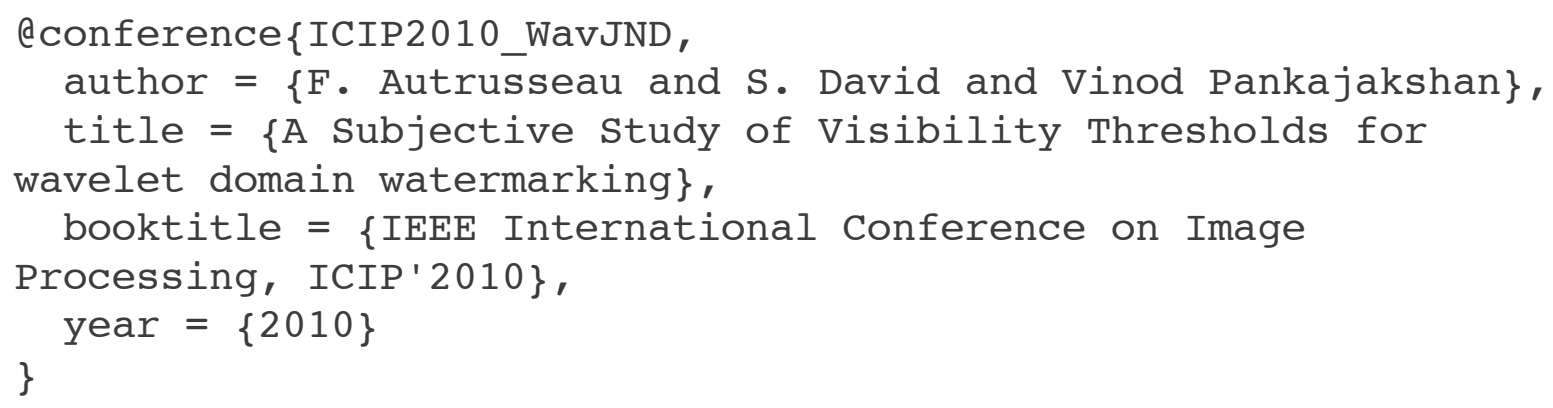




\title{
A SUBJECTIVE STUDY OF VISIBILITY THRESHOLDS FOR WAVELET DOMAIN WATERMARKING
}

\author{
Florent Autrusseau, Sylvain David, Vinod Pankajakshan \\ IRCCyN lab., University of Nantes \\ Rue Christian Pauc, 44306 Nantes, France
}

\begin{abstract}
Ensuring watermark invisibility in digital images is a challenging task. Most watermarking techniques empirically adjust a strength parameter in order to reach the best trade-off between invisibility and robustness. A target PSNR value is typically set in order to reach a defined quality level. Some watermarking techniques exploit local activity to increase the watermark strength in some specific images areas (edges, textures). In this work we study the visibility thresholds for wavelet domain multiplicative embedding using a watermarking oriented subjective experiment protocol. Thirty four observers were enrolled for a subjective experiment, and had to adjust the watermark strength in order to best define the visibility threshold for watermarking distortions. Three watermark embedding equations were tested in various wavelet sub-bands, and the optimal equation maximizing robustness was derived for every sub-band.
\end{abstract}

\section{INTRODUCTION}

In digital watermarking, various embedding domains like the DFT, DCT and DWT have been exploited for watermark insertion. Every embedding domain presents some advantages and drawbacks with respect to the most common watermarking requirements: invisibility, robustness and security. In particular, the wavelet domain has been a popular choice for watermark embedding, thanks to its multiresolution capabilities, a rather good Human Visual System modeling (HVS), and spatio-frequency localization. Effectively, most watermarking techniques empirically adjust the embedding strength in order to reach the best trade-off between invisibility, robustness and security. Basic HVS modeling for watermarking applications include luminance sensitivity, frequency sensitivity, and edge sensitivity [1]. The work in [2] also exploits contrast masking and the Watson distance for optimized Quantization Index Modulation (QIM) watermarking. The Noise Visibility Function (NVF) is commonly used to weight the watermark in the image areas having a higher perceptual embedding capacity [3]. The NVF basically uses the local variance as a weighting parameter. Pioneer works on image adaptive watermarking were conducted about ten years ago [4].
The authors exploited Watson's works on luminance sensitivity and contrast masking. They proposed two watermarking techniques operating either in the DWT domain or in the DCT domain. The watermark was weighted with a Just Noticeable Difference (JND) model and additively embedded in the transformed coefficients. Again, in these embedding techniques (either DCT or DWT based), the watermark energy was concentrated onto the high activity image areas (mostly edges and textures).

The work presented in this paper focuses on the watermark invisibility in wavelet domain embedding. There are two major differences between the previously cited watermarking techniques and the proposed work: 1) most watermarking schemes avoid embedding the watermark in the smooth areas of the image, as it supposedly can't stand any noise, in this work, we experimentally study the possibility of embedding a noise like watermark in smooth areas, and 2 ) in most of the cited works, some HVS features are considered in order to optimally embed the watermark in the image, whereas in this work, we experimentally determine the visibility threshold in wavelet sub-bands. Furthermore, as most of the subjective quality experiment procedures in the literature are designed for assessing coding distortions, we hereby setup a new subjective protocol which is particularly adapted to visibility threshold detection in watermarking.

Finally, in this work, we explore the appropriateness of using various watermarking embedding formulas. To the best of our knowledge, most (if not all) watermarking techniques use a single embedding equation, whatever frequency range is being watermarked (wavelet sub-band level for instance) and whatever is the wavelet coefficient value range. We hereby study the invisibility versus robustness trade-off in various wavelet sub-bands and using different watermark embedding equations.

This paper is structured as follows: Section 2 presents the embedding technique being used during the subjective test, Section 3 gives details on the experiment itself, we analyse the subjective data in Section 4 . In section 5 we evaluate the performances of the watermarking technique for various embedding equations and frequency bands. We finally conclude this contribution in Section 6 


\section{EMBEDDING EQUATIONS}

The goal of this work is to study the visibility thresholds in wavelet-domain watermarking using different embedding methods. The most commonly used wavelet-domain watermark scheme is the multiplicative embedding [5], in which the wavelet coefficients are modified as:

$$
Y_{i, j}(m, n)=X_{i, j}(m, n)+a \cdot\left|X_{i, j}(m, n)\right| \cdot W_{i, j}(m, n)
$$

where $X_{i, j}(m, n), W_{i, j}(m, n)$ and $Y_{i, j}(m, n)$ are respectively the $(m, n)$ location wavelet coefficient at sub-band $(i, j)$ for the host image, a zero mean, unit variance Gaussian distributed watermark and the marked image. The watermark strength, $a$, is usually determined empirically based on a target PSNR. We modified Equation 1 as:

$$
Y_{i, j}(m, n)=X_{i, j}(m, n)+a \cdot\left|X_{i, j}(m, n)\right|^{k} \cdot W_{i, j}(m, n)
$$

so that the watermark energy is spread differently along the edges depending on the parameter $k$. Three embedding equations are considered here, with $k=\{1,2,0.5\}$. The commonly used linear embedding scenario $(k=1)$, as well as a square function $(k=2)$ in order to further enhance the watermark strength in high amplitude wavelet coefficients (such as in [6]). Finally, the square root function $(k=0.5)$ increases the strength for low amplitude wavelet coefficients.

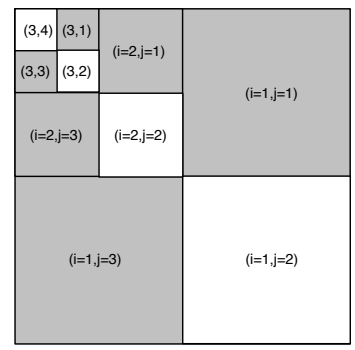

Fig. 1. Wavelet indexes used in Equation 2

A three level decomposition using CDF 9/7 bi-orthogonal wavelet is considered for watermark embedding. As the watermarks embedded in the diagonal sub-bands are more susceptible to lossy compression, those sub-bands are not considered in this study. The wavelet sub-bands used for watermark embedding are shown in gray shade in Figure 1.

\section{SUBJECTIVE EXPERIMENT}

Several experimental protocols are available for subjective quality assessment, see [7] for a brief description of standard experiments. In this study, we propose a new subjective protocol which is particularly suited for watermark visibility threshold detection. A subjective experimen 1 was set

\footnotetext{
${ }^{1}$ Source code: http://www.irccyn.ec-nantes.fr/ autrusse/jnd/
}

under normalized viewing conditions according to the ITUR BT.500-11 recommendations. The display monitor was an iMac with a $1920 \times 1080$ resolution, $50 \mathrm{~Hz}$, its minimum and maximum luminance were respectively $0.26 \mathrm{~cd} / \mathrm{m}^{2}$ and $213 \mathrm{~cd} / \mathrm{m}^{2}$. Thirty four observers with normal or corrected to normal acuity and normal colour vision were enrolled for the experiment. Since the subjective experiments are time consuming and tedious for the observers, some choices were made about the number of distortions and the number of input images in order to reduce the experiment duration. Five colour images of size $768 \times 512$ pixels, chosen from the Kodak image databas $\mathrm{f}^{2}$ were used in the experiment. The images were watermarked using the 3 embedding equations in the 6 selected wavelet sub-bands independently. For every trial, the observers had to stare at the watermarked images, displayed at a distance of six times the image height. The observers were asked to modify the $a$ parameter in Equation 2 in order to adjust the watermark strength just below the visibility threshold. The "up" and "down" arrows on the keyboard were used to increase/decrease $a$, and once the threshold set, the observers had to validate this selection with the "return" key. The initial value for $a$ was set to 0.00002 and the increment/decrement step was two (any stroke on the arrows multiplies or divides the coefficient by 2). Thirty images were displayed per session and the average session duration was about 20 minutes. Twelve observers collected the $a$ parameter for equation $k=1,12$ observers collected $a$ for $k=2$, and the remaining 10 observers collected the $a$ parameter for equation $k=0.5$. Using five input images watermarked in six wavelet sub-bands with three different embedding equations we have overall collected $1020 a$ parameters.

\section{SUBJECTIVE RESULTS ANALYSIS}

For each watermarked image and each embedding sub-band, the $a$ parameters given by the observers are averaged to get $\bar{a}$, which will be used in the following analysis. The watermark embedding at the visibility threshold for 6 wavelet sub-bands are plotted in Figures 2 and 3 for the "plane" and "farm" images respectively. The 3 rows of plots respectively represent high, middle and low frequency embedding. The insets in Figures 2 and 3 represents a zoom at the center of the plot. The $\mathrm{x}$-axis is the wavelet sub-band coefficient, and the y-axis is the watermark weighting function $\left(\bar{a} \times\left|X_{i, j}(m, n)\right|^{k}\right)$. The higher is the curve, the stronger is the watermark.

Evidently, the lower gets the watermark frequency, the lower is the embedding strength. In Fig. 2, the $\bar{a}$ parameters do not differ much across orientations of a given level and in Fig. $3, \bar{a}$ significantly differs across orientations. This is because the "farm" image has many high contrast horizontal edges, introducing larger wavelet coefficients in orientation 1 , consequently, the magnitude of the $\bar{a}$ parameter is restricted

\footnotetext{
${ }^{2}$ http://www.cipr.rpi.edu/resource/stills/kodak.html
} 

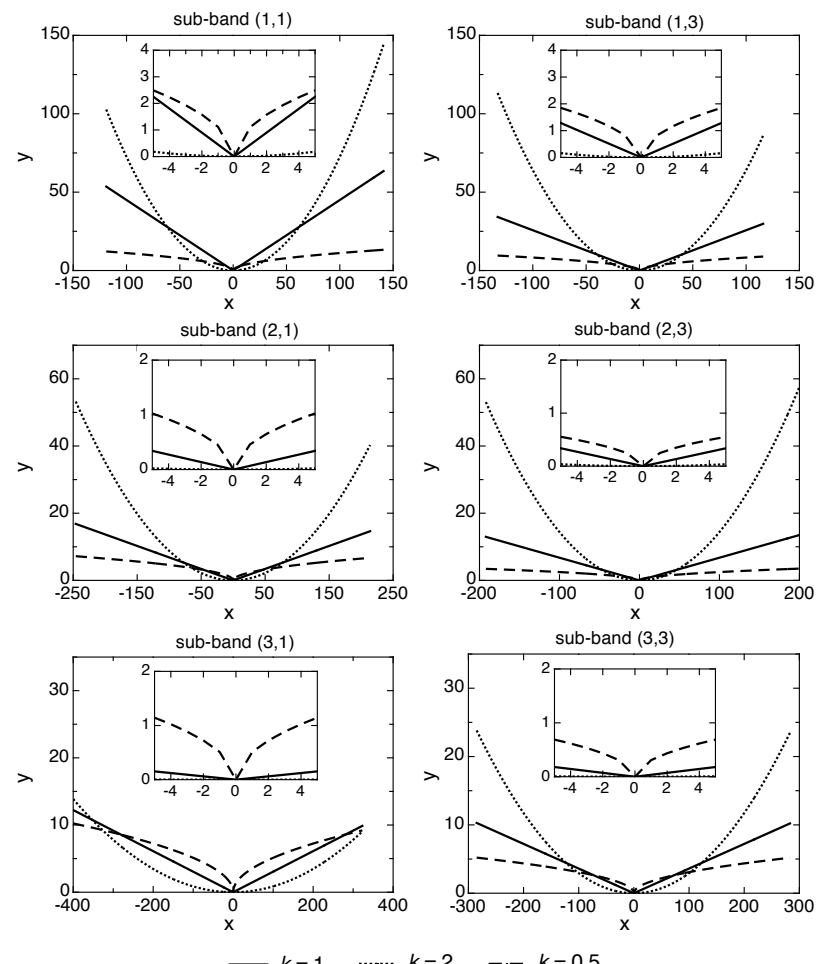

Fig. 2. Optimal watermark embedding depending on the three considered equations for image "plane"

by such high amplitude wavelet coefficients. Furthermore, we can notice on Figures 2 and 3 that the optimal choice of the embedding equation strongly depends on the embedding frequency. In lower frequency sub-bands (Level 3), using $k=0.5$ can achieve a quite strong watermark embedding, whereas for high and mid-frequency (Levels 1 and 2), using $k=1$ or 2 provides overall stronger watermarks. However, we can notice in the insets that for very low wavelets coefficients (having better robustness) using $k=0.5$ always provides stronger watermarks. An important observation from Figures 2 and 3 is that none of the 3 embedding equations is optimal by itself, and thus, combining the three equations might improve the watermark embedding.

Effectively, each equation reaches the visibility threshold in a certain range of wavelets coefficients. A stronger embedding is obtained using $k=0.5$ in the lowest wavelet coefficients, then, $k=1$ reaches the visibility threshold for higher coefficients, and using $k=2$ embeds stronger watermarks for highest coefficients. During the subjective experiment, it was observed that while increasing the watermark strength $a$, the visibility threshold was reached in distinct parts of the image depending on the embedding equation. When $k=1$ or 2 , the distortion first appeared on the image edges (having a higher energy in the wavelet sub-band), whereas for $k=0.5$, the distortions first appeared in the low luminance smooth areas.
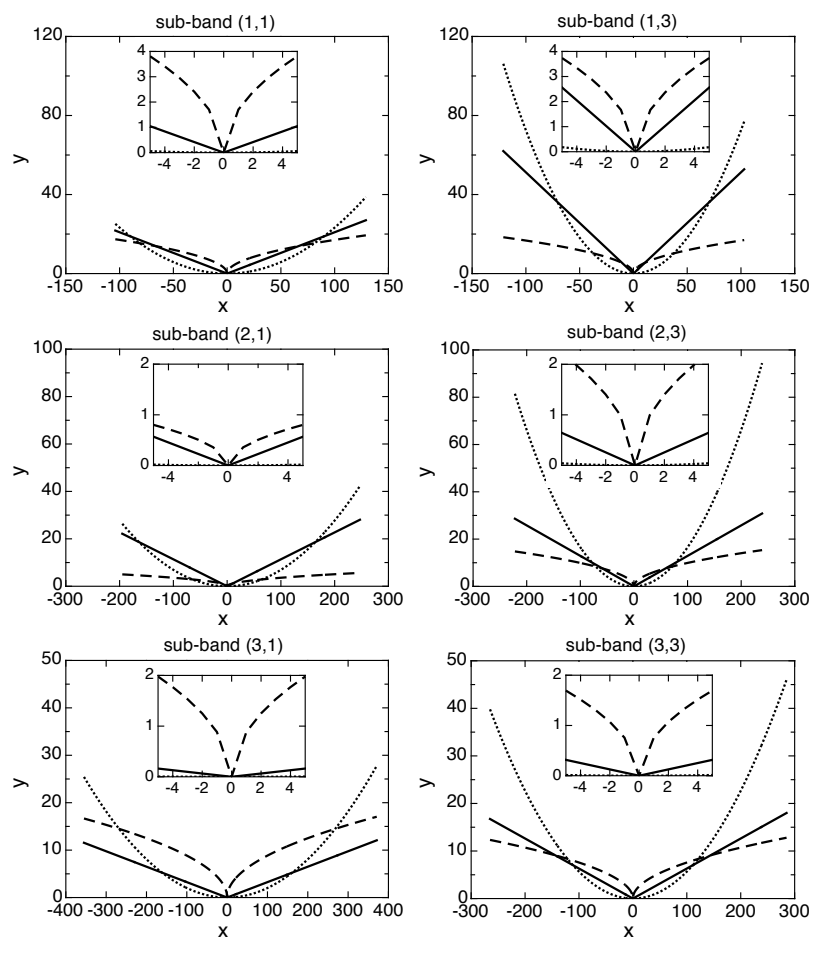

$-k=1 \quad \ldots \ldots k=2 \quad--k=0.5$

Fig. 3. Optimal watermark embedding depending on the three considered equations for image "farm"

\section{PERFORMANCES EVALUATION}

From the subjective experiment, we have collected the $\bar{a}$ parameters from the invisibility point of view. We now compare the robustness performances of different embedding equations to identify the optimal one in each sub-band. A set of 90 watermarked images was generated using Equation 2 with the $\bar{a}$ parameters. This work did not intend to provide an efficient watermarking technique, but rather to study the behavior of watermark embedding formulae at the visibility threshold. We thus used a simple non-blind detection technique, computing the correlation coefficient between $\left(\tilde{Y}_{i, j}(m, n)-X_{i, j}(m, n)\right)$ and $W_{i, j}(\mathrm{~m}, \mathrm{n}), \tilde{Y}_{i, j}(m, n)$ being the marked (and possibly distorted) wavelet sub-band. The detection threshold for each embedding equation in every wavelet sub-band was chosen as four times the maximum correlation coefficient corresponding to 200 wrong watermarks. The robustness performance was tested against JPG compression (quality factor from 70 to 10), JPEG 2000 (0.5bpp to $0.04 \mathrm{bpp}$ ), Gaussian noise addition (with variances ranging from 0.001 to 0.011 ), and various filtering methods (sharpening, Gaussian and median filtering). Figure 4 shows the correlation coefficients for the 35 tested attacks. The three curves stands for average high frequency bands (bands $(1,1)$ and $(1,3)$ in Fig. 1 represented with a solid line), medium frequency bands (bands $(2,1)$ and $(2,3)$ in dashed line) and 
low frequency bands (bands $(3,1)$ and $(3,3)$ in dotted line) and are averaged across the 5 input images. The thin horizontal lines represent the detection thresholds.

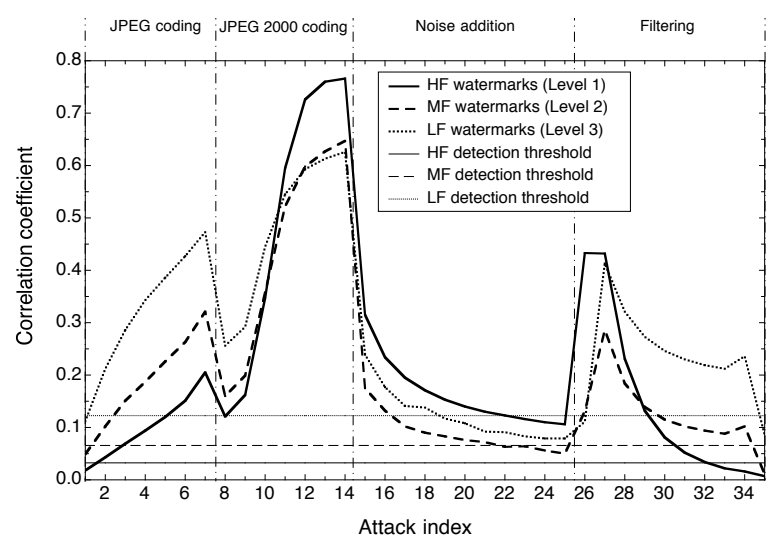

Fig. 4. Correlation coefficients against 35 attacks $(k=0.5)$

Due to space limitations, we only present the average detection performances within every frequency level. Percentages of correct detection are plotted on Fig. 5, which shows the detection performance for each of the three equations, and for every wavelet level. It can be observed that the square root embedding has the best robustness performance in all the levels, although the analysis of the subjective data showed overall a lower watermark strength in this scenario (Section 4). In particular, its performance is significantly better in the lowest frequency (level 3 ). Using $k=0.5$ actually embeds stronger watermarks in the low amplitude wavelet coefficients (see insets in Figures 2 and 3). Some attacks, such as coding or filtering, would more strongly modify the image edges, and thus a watermark embedded in the smooth areas, although of quite weak strength, might be more robust. Finally, it is interesting to notice that for all the embedding equations, the robustness decreases as the watermark embedding frequency decreases. This is probably because of the selected images, which have a quite low frequency content, and thus have a weak energy in the lowest wavelet levels, thereby limiting the embedding strength in such a frequency range.

\section{CONCLUSION}

A subjective protocol was proposed and tested for wavelet domain embedding. The observers were asked to modulate the watermark strength independently in 6 wavelet sub-bands. They were instructed to set the watermark strength just below the visibility threshold. The experiment showed that using a square root embedding equation provided better robustness for all wavelet levels. Although using $k=0.5$ presented a lower watermark strength during the subjective experiment its robustness performances are actually due to the smooth area embedding of this technique. In future works, we will try to

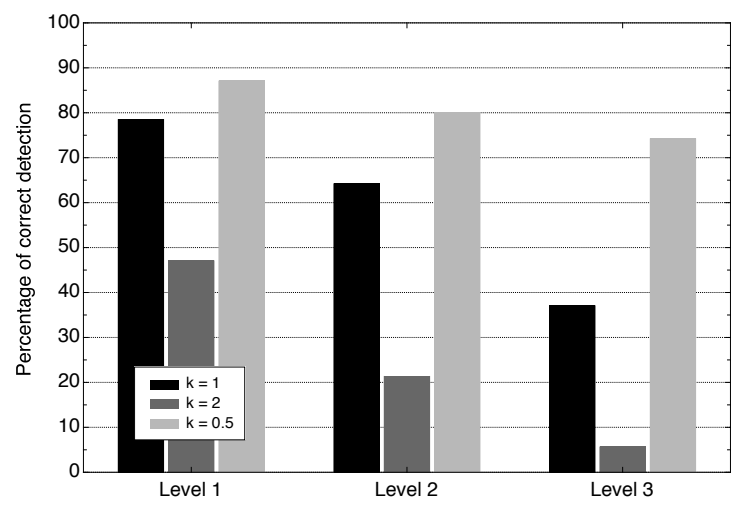

Fig. 5. Percentage of correct detection (against 35 attacks) for the three tested embedding equations

mathematically model the $a$ coefficient of the weighting function, so as to design a wavelet domain JND mask. We will furthermore study a possible combination of the three embedding equations in order to optimize the watermark strength in the whole wavelet coefficient range.

\section{REFERENCES}

[1] H. Qi, D. Zheng, and J. Zhao, "Human visual system based adaptive digital image watermarking," Signal Processing, vol. 88, no. 1, pp. 174-188, 2008.

[2] Q. Li and I. J. Cox, "Using perceptual models to improve fidelity and provide resistance to valumetric scaling for quantization index modulation watermarking," IEEE Trans. on Information Forensics and Security, vol. 2 (2), pp. 127-139, 2007.

[3] S. Voloshynovskiy, A. Herrigel, N. Baumgaertner, and T. Pun, "A stochastic approach to content adaptive digital image watermarking," in Proc. of the 3rd Intl. Workshop on Information Hiding, 2000, pp. 211-236.

[4] C. I. Podilchuk and W. Zeng, "Image adaptive watermarking using visual models," IEEE Journal on Selected Areas in Comm., vol. 16, (4), pp. 525-539, 1998.

[5] I.J. Cox, J. Kilian, F.T. Leighton, and T. Shamoon, "Secure spread spectrum watermarking for multimedia," IEEE Trans. on Image Proc., pp. 1673-87, 1997, 6(12).

[6] X. Xia, C. G. Boncelet, and G. R. Arce, "Wavelet transform based watermark for digital images," Optics Express, vol. 3(12), pp. 497-511, 1998.

[7] P. Le Callet, F. Autrusseau, and P. Campisi, Multimedia Forensics and Security, chapter IX, pp. 164-193, Idea Group Publishing, 2008. 